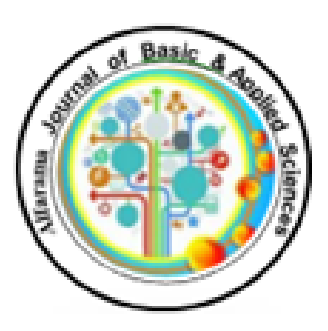

ISSN 2682-275X

Faculty of Science Port Said University http://sci.psu.edu.eg/en/

January 2020, Volume 1, Issue $1 \quad$ DOI:10.21608/AJBAS.2019.19064.1003

Submitted: 09-11-2019

Accepted: 01-12-2019

Pages: 22-28

\title{
MODELLING THE CREATION OF SURFACE NANO-STRUCTURES USING TEST CHARGE APPROACH
}

\author{
Ibrahiem A Elsheikh ${ }^{1,2}$, Waleed M Moslem ${ }^{1,2, *}$, Amr El-Zant ${ }^{2}$ \\ ${ }^{1}$ Department of Physics, Faculty of Science, Port Said University, Port Said, Egypt \\ ${ }^{2}$ Centre for Theoretical Physics, The British University in Egypt (BUE), El-Shorouk city, Cairo, Egypt \\ ${ }^{*}$ Corresponding author: Wmmoslem@sci.psu.edu.eg
}

\begin{abstract}
Nowadays, surface nano-structures has become an important technological method that plays an important role in the electronic devices, as it has a very remarkable feature is that they are used in etching without using chemicals. Recently, the plasma expansion model was used to describe what happens in the creation of surface nano-structures in materials. In this paper, another model to describing the creation of surface materials like $\mathrm{SiO}_{2}$ has been introduced, using a test charge approach. The basic equations describing the $\mathrm{SiO}_{2}$ are reduced to one evolution equation characterizing the wakefield electric potential of a moving test charged particle. The profile of the wakefield potential is examined with different plasma parameters. It has been found that the wakefield potential decreases for different values of negative and positive ions densities. Also, we deduced that the fast test charge creates high wakefield potential behind it. Thus, they drag more charges to follow it creating the surface nano-structure.
\end{abstract}

Keywords:

Test charge; Surface nano-structure; Plasma modeling.

\section{INTRODUCTION}

Plasma expansion [1,2], has attracted attention as a means of explaining the increase of temperature in devices. Research in the field began in 1945 by Landau [3] and since then has developed considerably. Plasma expansion is now ubiquitously observed in experimental setups, where the required high tremperatures are reached via laser irradiation. Driven by these temperatures, the plasma expands 
into the surrounding vaccum. Plasma expansion is also studied in astrophysical systems, such as planetary rings, solar wind, and in inert objects as Titan moon and Venus [4, 5, 6, 7].

In the last two decades, a new method has been used to create surface nano-structures in different semiconductors such as $\mathrm{SiO} 2$ [8], $\mathrm{ZnO}$ [9] and $\mathrm{Si}$ [10]. Many applications depend on creating such structures in various materials (e.g. Refs $[11,12,13,14])$. Surface nano-structures have thus come to constitute a technological tool that plays an important role in the design of electronic devices; a tool that is particularly appealing, given that their use has the most important advantage of rendering it possible to achieve etching without using chemicals.

In recent years, the plasma expansion model was used to describe the creation of surface nanostructures in materials. Moslem and El-Said [15], for example, investigated the creation of surface nano-structures by highly charged ions; in particular, they used the approach to derive equations describing the formation mechanism of the surface nano-hillocks pumped by HCIs. The goal of this work is to explain the plasma expansion in surface nano-structures of $\mathrm{SiO}_{2}$ single crystal using an alternative approach; namely, the test charge technique. Indeed, the used model in Refs. $[9,15]$ is not the unique model for plasma acceleration, although it is nonlinear model, so we suggest a test charge approach as a possible model that can explain the plasma dragging into space. The test charge approach is based on a plasma linear theory, which may introduce an alternative mechanism for the creation of surface nano-structures. The test charge model goes back at least to 1985 when it was presented by Nambu and Akama [16]. Later, many theoretical and experimental works used this model in ordinary, dusty, and quantum plasmas (e.g. Refs [17, 18, 19]). In the present context, we use a test charge method, involving a moving ion (i.e. $\mathrm{Si}$, and $\mathrm{O}$ ) surrounded by electrons with Maxwellian velocities, to describe the possible mechanism of the surface nano-structures formation.

\section{THEORETICAL MODEL}

The formation of surface nano-hillocks using a deposited energy by the HCIs may be sufficient to produce a plasma in the ion impact region that can expand above the surface. For $\mathrm{SiO}_{2}$ case, the plasma consists of negative ions " $\mathrm{O}^{-}$, positive ions " $\mathrm{Si}^{+}$", as well as electrons. We suggest a collisionless plasma since the collision time scale is large compared to the ion-acoustic wave time scale and the expansion of the nano-plasma hillocks happened in tens of picosecond. Also, we consider a classical plasma model although the ion density is high, but it lies in the border of classical and quantum plasma regime. In the future, we will consider the quantum plasma model to have a comprehensive vision about the possible plasma models. Using the linearized Vlasov equation, we can describe the dynamics of $\mathrm{SiO}_{2}$ fluid model as

$$
\left(\partial_{t}+\mathbf{V} \cdot \nabla\right) f_{j 1}+\frac{q_{j}}{m_{j}} \mathbf{E}_{1} \cdot \nabla_{\mathbf{v}} f_{j 0}(\mathbf{V})=0
$$

where $f_{j 1}\left(\ll f_{j 0}\right)$ is the perturbed (unperturbed) particle distribution function, $q_{j}$ and $m_{j}$ are the charge and mass, respectively. $\nabla=\hat{\mathbf{e}}_{x} \partial / \partial X+\hat{\mathbf{e}}_{y} \partial / \partial Y+\hat{\mathbf{e}}_{z} \partial / \partial Z$, and $\nabla_{\mathbf{v}}=\hat{\mathbf{e}}_{x} \partial / \partial V_{x}+\hat{\mathbf{e}}_{y} \partial / \partial V_{y}+$ $\hat{\mathbf{e}}_{z} \partial / \partial V_{z}$, where $\hat{\mathbf{e}}_{x}, \hat{\mathbf{e}}_{y}$, and $\hat{\mathbf{e}}_{z}$ are the unit vectors along the $\mathrm{x}$-, $\mathrm{y}$-, and z-axes, respectively. Here, $\mathbf{E}_{1}\left(=-\nabla \varphi_{1}\right)$ is the induced electric field with the electrostatic potential $\varphi_{1}$. Equation (1) is coupled through Poisson equation by the perturbed distribution function $f_{j 1}$, as

$$
\begin{aligned}
\nabla^{2} \varphi_{1} & =-4 \pi\left(\rho_{\text {plasma }}+\rho_{\text {test }}\right) \\
& =-4 \pi \sum_{j} q_{j} \int f_{j 1} d \mathbf{V}-4 \pi Q_{p} \delta\left(\mathbf{r}-\mathbf{V}_{p} t\right),
\end{aligned}
$$

where $\rho_{\text {plasma }}$ represents the charge density of the plasma particles, i.e., positive ions, negative ion species, and electrons, $\rho_{\text {test }}$ is the test charge density, $Q_{p}$ is the charge of the test particle propagating 
through the plasma with a constant velocity $\mathbf{V}_{p}$ along the z-axis. Here, $\delta\left(\mathbf{r}-\mathbf{V}_{p} t\right)$ is the threedimensional Dirac delta function. The test charge can be assumed to be at rest at origin in a steady state plasma for $\mathbf{V}_{p}=0$.

Applying space-time Fourier transformations, Eqs. (1) and (2) can be combined as

$$
\tilde{\varphi}_{1}(\mathbf{k}, \omega)=\frac{8 \pi Q_{p} \delta\left(\omega-\mathbf{k} \cdot \mathbf{V}_{p}\right)}{k^{2} \varepsilon(k, \omega)}
$$

where $\omega$ and $\mathbf{k}$ are the wave frequency and wave vector, respectively. $\varepsilon(k, \omega)$ is the dielectric constant given by

$$
\varepsilon(k, \omega)=1+\sum_{j} \frac{K_{D j}^{2}}{k^{2}} W\left(C_{j}\right) .
$$

In Eq. (4), $W\left(C_{j}\right)$ is the plasma dispersion function which is given by

$$
W\left(C_{j}\right)=(1 / \pi)^{1 / 2} \int_{-\infty}^{\infty} \frac{q \exp \left(-q^{2} / 2\right)}{q-C_{j}} d q,
$$

where $C_{j}=\omega / k_{z} V_{t j}, V_{t j}$ is the thermal speed, and $K_{D j}$ is the Debye wavenumber which is actually the inverse of the Debye length $\lambda_{D e}\left(=\sqrt{T_{e} / 4 \pi e^{2} n_{e}^{(0)}}\right)$.

For ion-acoustic waves, we have $V_{t, 1,2} \ll \frac{\omega}{k} \ll V_{t e}$, where 1 and 2 refer to $\mathrm{Si}^{+}$and $O^{+}$, respectively. Therefore, the plasma dispersion function gives $W\left(C_{e}\right)=1, W\left(C_{i}\right)=-1 / 2 C_{i}^{2}, W\left(C_{1}\right)=-1 / 2 C_{1}^{2}$, and $W\left(C_{2}\right)=-1 / 2 C_{2}^{2}$, for electrons, positive ions, and negative ions, respectively. Introducing the plasma dispersion functions with Eq. (4), the dielectric constant reads

$$
\frac{1}{\varepsilon(k, \omega)}=\frac{k^{2} \lambda_{D e}^{2}}{k^{2} \lambda_{D e}^{2}+1}\left(1+\frac{\omega_{k}^{2}}{\omega^{2}-\omega_{k}^{2}}\right)
$$

with

$$
\omega_{k}^{2}=\frac{\left(\omega_{p S i}^{2}+\omega_{p O}^{2}\right)\left(k^{2} \lambda_{D e}^{2}\right)}{1+k^{2} \lambda_{D e}^{2}}
$$

is the dispersion relation of the ion-acoustic waves which the positive ion is modified by the negative ion plasma frequency $\omega_{p o}=\left(4 \pi e^{2} n_{1(2)}^{(0)} / m_{1(2)}\right)^{1 / 2}$, and $\omega_{p S i}=\left(4 \pi e^{2} n_{i}^{(0)} / m_{i}\right)^{1 / 2}$ where, $m_{1,2}$ is the masses of positive ion and negative ions, $\lambda_{D e}=\left(T_{e} / 4 \pi e^{2} n_{e}^{(0)}\right)^{1 / 2}$ is the electron Debye length.

Taking the inverse space-time Fourier transformation to (3), we can express the electrostatic potential at an arbitrary position $\mathrm{r}$, as

$$
\varphi_{1}(\mathbf{r}, t)=\frac{Q_{p}}{2 \pi^{2}} \int \frac{d \mathbf{k}}{k^{2}} \frac{\exp \left[i \mathbf{k} \cdot\left(\mathbf{r}-\mathbf{V}_{p} t\right)\right]}{\varepsilon\left(k, \mathbf{k} \cdot \mathbf{V}_{p}\right)}
$$

Substituting (5) into (7), the potential, becomes

$$
\varphi_{D}(\mathbf{r}, t)=\frac{Q_{p}}{2 \pi^{2}} \int d \mathbf{k}\left(\frac{\lambda_{D e}^{2}}{k^{2} \lambda_{D e}^{2}+1}\right) \exp \left[i \mathbf{k} \cdot\left(\mathbf{r}-\mathbf{V}_{p} t\right)\right] .
$$

We shall use the spherical polar coordinates to simplify Eq. (8), by using $\mathbf{k}=\left(k \sin \theta_{k} \cos \varphi_{k}\right.$, $\left.k \sin \theta_{k} \sin \varphi_{k}, k \cos \theta_{k}\right), \quad \mathbf{r}=\left(r \sin \theta_{r} \cos \varphi_{r}, r \sin \theta_{r} \sin \varphi_{r}, r \cos \theta_{r}\right), \quad$ and $\mathbf{V}_{p}=\left(0,0, V_{p}\right), \quad$ so that 
$\mathbf{k} \cdot\left(\mathbf{r}-\mathbf{V}_{p} t\right)=k \rho \sqrt{1-\mu^{2}} \cos \varphi_{k}+k \mu Z$, where $\rho\left(=r \sin \theta_{r}\right)$ and $Z\left(=r \cos \theta_{r}-V_{p} t\right)$ are the radial and axial distances of a test charge from an observation point $\mathbf{r}, \mu=\cos \theta_{k}$, and $\sin \theta_{k}=\sqrt{1-\mu^{2}}$. After some algebraic manipulations, the modified Debye-Hückel potential is (further details can be found in Ref. [20])

$$
\varphi_{D}=\frac{Q_{p}}{r} \exp \left(-\frac{r}{\lambda_{D e}}\right)
$$

where $r=\left(\rho^{2}+Z^{2}\right)^{1 / 2}$.

To derive the wake potential $\phi_{W}$, it convenient to use the cylindrical coordinates, where $\mathbf{k} \cdot\left(\mathbf{r}-\mathbf{V}_{p} t\right)=$ $k_{\perp} \rho \cos \varphi_{k}+k_{\|} \xi$ and $d \mathbf{k}=k_{\perp} d k_{\perp} k_{\|} d \varphi_{k}$ to obtain

$$
\varphi_{W}=\frac{2 Q_{p}}{Z}\left[1+\frac{\left(\omega_{p S i}^{2}+\omega_{p O}^{2}\right)}{V_{p}^{2}} \lambda_{D e}^{2}\right]\left[1-\frac{\left(\omega_{p S i}^{2}+\omega_{p O}^{2}\right)}{V_{p}^{2}} \lambda_{D e}^{2}\right]^{-1} \cos \left(\frac{\left(\omega_{p S i}^{2}+\omega_{p O}^{2}\right)^{1 / 2}}{V_{p}} Z\right)
$$

Equation (10) is a test charge wakefield potential propagating with a fixed speed along the z-axis. It is noted that Eq. (10) contains the dynamics of the negative ion species $\left(\mathrm{O}^{-}\right)$. Neglecting the negative ions, we have $\omega_{p O}^{2}=0$, and thus the wakefield potential, from (10), is reduced to an expression including the dynamics of the positive ions only.

\section{RESULTS AND DISCUSSION}

We will discuss the characteristics of Debye and wakefield potentials in the surface of nano-structure. We normalized Eqs. (9) and (10), where the potentials are normalized by $q_{1} / \lambda_{D}$, the velocities by

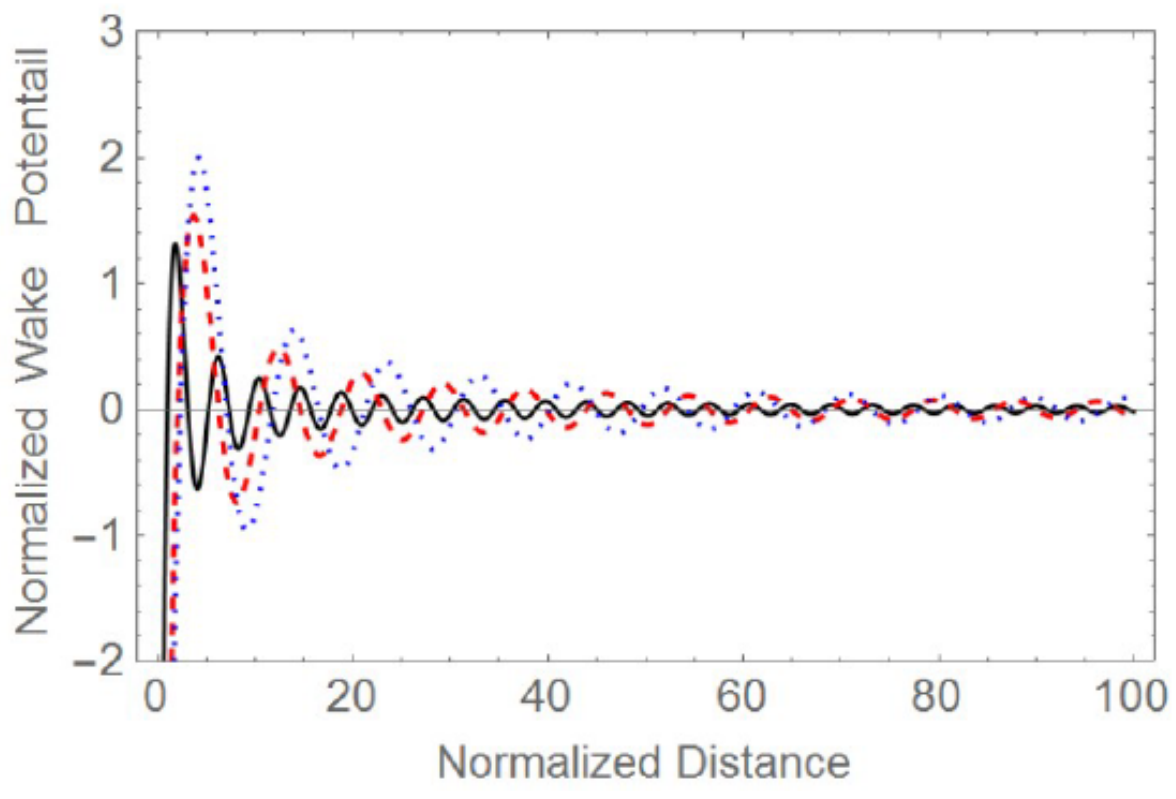

Figure 1: (Color online) The normalized wakefield potential is depicted vs. the normalized axial distance for various values of positive and negative ion density $n_{S i}=1.1 \times 10^{21} \mathrm{~cm}^{-3}$ (solid-black curve), $n_{S i}=1.5 \times 10^{21} \mathrm{~cm}^{-3}$ (dashed-red curve), $n_{O}=0.1 \times 10^{21} \mathrm{~cm}^{-3}$ (dashed-blue curve). 
$V_{p}=\omega_{p d} \lambda_{D}$ and spatial distances by effective Debye length $\lambda_{D}$. The ions number density $n_{S i, O} \approx$ $1 \times 10^{21} \mathrm{~cm}^{-3}$.

We can describe the Debye and wake potentials around a test charge with the aid of the above normalized parameters. It is cleared that the normalized Debye potential reduces exponentially with the normalized total distance from an observation point $r$, so we did not include this figure here. Figures 1 and 2 display the profile of the normalized wakefield potential $\varphi_{W}$ with the normalized axial distance $Z$. In general, the wakefield is produced behind the test charge and it damps with the axial distance $Z$. Its maximum value is important to predict an electric potential near the charge. Figure (1) show that how the wakefield potential magnitude changes with the normalized distance $Z$. Initially, it has high magnitude and then becomes small with distance. Figure 2 presents the effect of the phase velocity on the system. The speed is assumed to be greater than the ion-acoustic velocity. The latter can be calculated from its usual mathematical expression. The wake potential field increases with the increase of the phase velocity. On the other hand, a fast test charge creates high wake potential behind it to drag more charges to follow it. It is interesting to mention here that if the speed of the test charge is less than the ion-acoustic velocity, then the wakefield potential will not form because of the test charge will be behind the wave front.

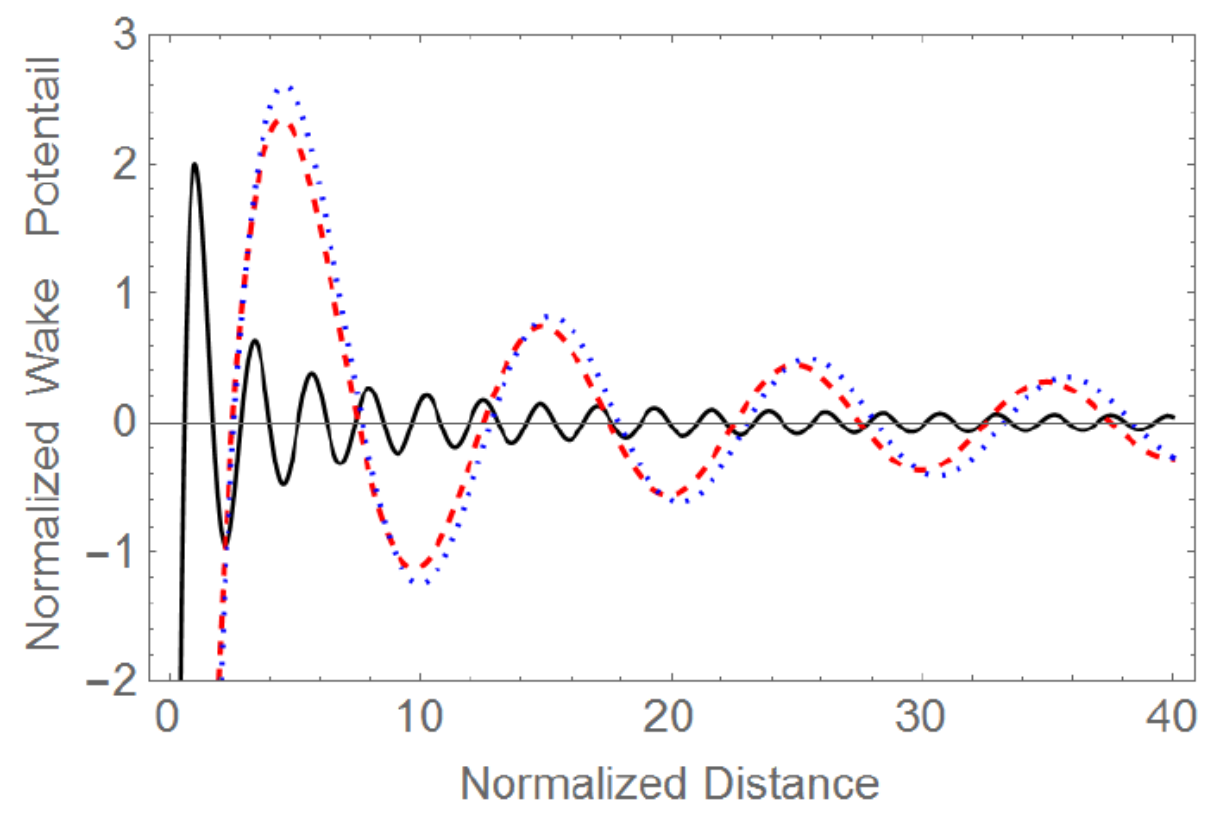

Figure 2: (Color online) The normalized wakefield potential is depicted vs. the normalized axial distance for various values of positive and negative ion density $V_{p}=1 \times 10^{6} \mathrm{~cm} / \mathrm{s}$ (solid-black curve), $V_{p}=4.5 \times 10^{6} \mathrm{~cm} / \mathrm{s}$ (dashed-red curve), $V_{p}=1.7 \times 10^{7} \mathrm{~cm} / \mathrm{s}$ (dashed-blue curve).

\section{SUMMARY}

We applied a test charge model to describe the creation of surface nano-structure for $\mathrm{SiO}_{2}$. It has been noticed that the normalized wakefield potential decreases with for different values of negative and positive ions densities. Also we deduced that the fast test charge creates high wake potential behind it, thus they drag more charges to follow it that may lead to the creation of surface nano-structues. Therefore, we introduce a possible mechanism to form surface nano-hillocks in $\mathrm{SiO}_{2}$. 


\section{References}

[1] U. R. I. Samir, K. H. Wright, Jr., and N. H. Stone, "The expansion of a plasma into a vacuum: Basic phenomena and processes and applications to space plasma physics", Rev. Geophys. Space Phys., vol. 21, no. 7, pp. 1631-1646, 1983.

[2] B. Doggett and J. G. Lunney, "Expansion dynamics of laser produced plasma", J. Appl. Phys., vol. 109, no. 9, pp. 3304, 2011.

[3] L. D. Landau, E. M. Lifshitz, M. Sploshnykh Sred, "Mechanics of Continuous Media", J. Appl. Math. Mech., vol. 26, no, 5, pp. 1293-1309, 1954.

[4] M. S. Tiscareno and C. D. Murry, "Plentary Rings System", United Kingdom TJ International Ltd, Padstow, Cornwall, pp 517-530, 2018.

[5] A. V. Artemyev, V. Angelopoulos, and J. M. McTiernan, "Near-Earth solar wind: plasma characteristics from ARTEMIS measurements", J. Geophys. Res. Space Sci., vol 123, no.12, pp. 9955-9962, 2018.

[6] S. Salem, W. M. Moslem, and A. Radi, "Expansion of Titan atmosphere", Phys. Plasmas, vol. 24, no. 5, pp 2901, 2017.

[7] H. Perez-de-Tejada, R. Lundin, Y. Futaana, T. L. Zhang, "Properties of planetward ion ows in Venus magnetotail", Icarus, vol. 274, no. 24, pp. 73-82, 2016.

[8] A. S. El-Said, "Nanostructures created in $\mathrm{SiO} 2$ surface: A comparison between the impingement by slow highly charged ions and by swift heavy ions", Nucl. Instrum. Meth. B, vol. 282, pp. 63, 2012.

[9] A. S. El-Said, W. M. Moslem, and M. Djebli, "Surface nanostructuring by ion-induced localized plasma expansion in zinc oxide", Appl. Phys. Lett., vol. 104, pp. 231609, 2014.

[10] M. Tona and S. Ohtani, "Surface processes of highly charged ions", J. Phys: Conf. Ser., vol. 185, pp. 012046, 2009.

[11] O. Y. Bozkurt, O. Ozbek, and A. R. Abdo, "The Effects of Nanosilica on Charpy Impact Behavior of Glass/Epoxy Fiber Reinforced Composite Laminates" Period. Engin. Nat. Sci. vol. 5, no. 3, pp. 322, 2017.

[12] O. Inayatullah and V. Sinnasamy, Condition Monitoring of the Uncoated Carbide Cutting Tool in Turning Process of the Aluminum Alloy 6061 via Vibration, Period. Engin. Nat. Sci. vol. 5, no. 3, pp. 298, 2017.

[13] J. Sutkovic and J. Jasarevic, "A review on Nanoparticle and Protein interaction in biomedical applications" Period. Engin. Nat. Sci. vol. 4, no. 2, pp. 34, 2016.

[14] A. S. El-Said, "Nanostructures created in $\mathrm{SiO}_{-} 2$ surface: A comparison between the impingement by slow highly charged ions and by swift heavy ions", Nucl. Instrum. Meth. B, vol. 282, pp. 63, 2012.

[15] S.A.Morsi, W.M.Moslem, and S.K.El-Labany "Interpretation of localized surface nanostructures" Period. Engin. Nat. Sci. vol. 7, no. 2, pp. 881, 2019. 
[16] M. Nambu and H. Akama "Attractive potential between resonant electrons" phys. Fluid. vol. 28, no.7, pp. 2300, 1985.

[17] W.M.Moslem, R.E.Tolba, and S.Ali "Potentials of a moving test charge during the solar wind interaction with dusty magnetosphere of Jupiter" Phys. Scr. vol. 94, no. 7, pp. 7, 2019.

[18] S. Ali and P. K. Shukla "Potential distributions around a moving test charge in quantum plasmas" Phys. Plasmas. vol. 13, no. 10, pp. 102, 2006.

[19] P. K.Shukla and A.A.Mamun "Introduction to dusty plasma physics" IOP.London, 2002, and references there in.

[20] W. M. Moslem, R. Sabry, and P. K. Shukla, "The optimum shielding around a test charge in plasmas containing two negative ions", J. Plasma Phys. vol. 77, pp. 663, 2011. 